\title{
Metabolism of oxycodone in human hepatocytes from different age groups and prediction of hepatic plasma clearance
}

\author{
Timo Korjamo ${ }^{1 \dagger}$, Ari Tolonen ${ }^{2}$, Veli-Pekka Ranta ${ }^{3}$, Miia Turpeinen $^{4 *}$ and Hannu Kokki ${ }^{5}$ \\ Novamass Ltd., Oulu, Finland \\ ${ }^{2}$ Admescope Ltd., Oulu, Finland \\ ${ }^{3}$ School of Pharmacy, University of Eastern Finland, Kuopio, Finland \\ ${ }^{4}$ Department of Pharmacology and Toxicology, Institute of Biomedicine, University of Oulu, Oulu, Finland \\ ${ }^{5}$ Department Anaesthesiology, Kuopio University Hospital and School of Medicine, University of Eastern Finland, Kuopio, Finland
}

\section{Edited by:}

Patrick Maurel, UMR-U632, France

Reviewed by:

Moshe Finel, University of Helsinki, Finland

Nils Tore Vethe, Oslo University Hospital, Norway

Stanislav Yanev, Institute of

Neurobiology, Bulgaria

*Correspondence:

Miia Turpeinen, Department of Pharmacology and Toxicology,

Institute of Biomedicine, University of

Oulu, Aapistie 5B, 90220 Oulu,

Finland.

e-mail:miia.turpeinen@oulu.fi

${ }^{\dagger}$ Present address:

Timo Korjamo, Orion Corporation,

Orion Pharma, Espoo, Finland.
Oxycodone is commonly used to treat severe pain in adults and children. It is extensively metabolized in the liver in adults, but the maturation of metabolism is not well understood. Our aim was to study the metabolism of oxycodone in cryopreserved human hepatocytes from different age groups ( 3 days, 2 and 5 months, 4 years, adult pool) and predict hepatic plasma clearance of oxycodone using these data. Oxycodone $(0.1,1$, and $10 \mu \mathrm{M})$ was incubated with hepatocytes for $4 \mathrm{~h}$, and $1 \mu \mathrm{M}$ oxycodone also with CYP3A inhibitor ketoconazole $(1 \mu \mathrm{M})$. Oxycodone and noroxycodone concentrations were determined at several time points with liquid chromatography-mass spectrometry. In vitro clearance of oxycodone was used to predict hepatic plasma clearance, using the well-stirred model and published physiological parameters. Noroxycodone was the major metabolite in all batches and ketoconazole inhibited the metabolism markedly in most cases. A clear correlation between in vitro oxycodone clearance and CYP3A4 activity was observed. The predicted hepatic plasma clearances were typically much lower than the published median total plasma clearance from pharmacokinetic studies. The data suggests that there are no children-specific metabolites of oxycodone. Moreover, CYP3A activity seems to be the major determinant in metabolic clearance of oxycodone regardless of age group or individual variability in hepatocyte batches.

Keywords: drug metabolism, hepatocytes, oxycodone, cytochrome P450, pediatric model, in vitro

\section{INTRODUCTION}

Oxycodone (7,8-dihydro-14-hydroxycodeinone) is a commonly prescribed opioid for severe pain, and during the last years its use has surpassed that of morphine in several countries (International Narcotics Control Board, 2010). Oxycodone is extensively metabolized in the liver and only ca. $10 \%$ of the dose is excreted unchanged in urine in adults (Pöyhiä et al., 1992; Kirvelä et al., 1996; Lalovic et al., 2006), but the majority of the metabolites are excreted in urine (Lalovic et al., 2006). The principal metabolic pathway is CYP3A-mediated $\mathrm{N}$-demethylation to noroxycodone (Lalovic et al., 2004, 2006). Another important route is CYP2D6-mediated $O$-demethylation to oxymorphone. A large portion of noroxycodone and oxymorphone is further metabolized or conjugated, e.g., noroxymorphone is formed mainly via $O$-demethylation of noroxycodone by CYP2D6. Oxymorphone and noroxymorphone are active metabolites, but they probably do not contribute markedly to the central opioid effects since their plasma concentrations are significantly lower than that of parent compound (Heiskanen et al., 1998; Lalovic et al., 2006; Lemberg et al., 2010). Furthermore, these metabolites are less lipophilic than oxycodone, a characteristic that may limit their uptake into the brain (Lalovic et al., 2006).

There seems to be no major differences in the pharmacokinetics of oxycodone between children and adults (Olkkola et al., 1994;
Kokki et al., 2004, 2006), and a weight-based dose can be used for children and infants aged over 6 months (El-Tahtawy et al., 2006). On the contrary, the dose titration for infants younger than 6 months must be cautious because the inter-individual variation in the clearance and elimination of oxycodone is significant and the clearance could be very low in some of the youngest subjects (Pokela et al., 2005).

Although oxycodone has been in clinical use for over 90 years (Falk, 1917), its metabolic pathways in infants and children are not sufficiently understood. In addition, FDA guidance recommends to study pharmacokinetic phenomena also in pediatric models such as in vitro metabolic studies with pediatric preparations (FDA Guidance for Industry, 1998). In the present study, we measured the maturation of oxycodone metabolism in vitro using human hepatocytes from different age groups. In addition, we predicted the hepatic plasma clearance of oxycodone based on these data and estimated the accuracy of the prediction.

\section{MATERIALS AND METHODS MATERIALS}

Oxycodone, noroxycodone, oxymorphonem, and noroxymorphone were obtained from Cerilliant (Round Rock, TX, USA), and ketoconazole from Sigma (St. Louis, MO, USA). Highperformance liquid chromatographic (HPLC) grade methanol and 
acetonitrile were obtained from Merck (Darmstadt, Germany). Ammonia was obtained from BDH Laboratory Supplies (Poole, UK). Other chemicals were obtained from Sigma (St. Louis, MO, USA) and were of the highest purity available. Water (ultra pure, 18.2 M $\Omega$ ) was freshly prepared with Direct-Q3 purification system (Millipore Oy, Espoo, Finland). Human cryopreserved hepatocytes were obtained from BD Biosciences (Franklin Lakes, NJ, USA), Celsis (Brussels, Belgium), and Invitrogen (Carlsbad, CA, USA). The donors were 3-day, 5-month, and 4-year-old Caucasian females, a 2-month-old Caucasian male, and a pool of 20 adults (pools of 10 women and 10 men were combined), mostly Caucasians. The 3-day-old donor had received phenobarbital that is a potent inducer of CYP3A and several other CYP enzymes.

\section{IN VITRO INCUBATION OF OXYCODONE WITH CRYOPRESERVED HEPATOCYTES}

Oxycodone concentrations in the incubations $(0.1-10 \mu \mathrm{M})$ were chosen to be close to the clinical plasma concentrations of oxycodone (typically $<0.3 \mu \mathrm{M}$ ) and much lower than the mean $K_{\mathrm{m}}$ values for CYP3A-mediated noroxycodone formation $(600 \mu \mathrm{M})$ and CYP2D6-mediated oxymorphone formation $(130 \mu \mathrm{M})$ in human liver microsomes (Lalovic et al., 2004). The hepatocytes were thawed and suspended according to Celsis protocol. Briefly, tubes were rapidly thawed, cells suspended into Celsis InVitro GRO HT-medium, and centrifuged $(50 \times g, 5 \mathrm{~min})$. Cells were resuspended into Celsis InVitro GRO HI-medium. The cell density and viability were determined by trypan blue exclusion method. The viabilities of the individual donor cells varied from 75 to $98 \%$. Cells were diluted into InVitro GRO HI to two million viable cells per milliliter. Oxycodone was dissolved into DMSO at $2 \mathrm{mM}$ concentration and diluted into InVitro GRO HI to concentrations 0.2 , 2 , and $20 \mu \mathrm{M}$. Oxycodone solution $(175 \mu \mathrm{l})$ was applied to 48 -well tissue culture plate in a single well. An equal volume of hepatocyte suspension was added to the well yielding final hepatocyte concentration of one million viable cells per milliliter in $0.1,1$, or $10 \mu \mathrm{M}$ oxycodone solution. Plates were incubated at $37^{\circ} \mathrm{C}$ in humidified CO2-incubator with $600 \mathrm{rpm}$ orbital stirring. Samples of $50 \mu \mathrm{l}$ were taken at $0,30,60,120$, and $240 \mathrm{~min}$ from the well and suspended into $50 \mu \mathrm{l}$ of cold acetonitrile. The incubations with $1 \mu \mathrm{M}$ oxycodone were conducted also with $1 \mu \mathrm{M}$ ketoconazole. Two replicates of each experiment were conducted. Samples were stored at $-20^{\circ} \mathrm{C}$ until analysis.

\section{ESTIMATION OF OXYCODONE FREE FRACTION IN HEPATOCYTE INCUBATION}

The passive accumulation into hepatocytes was studied with cells that were kept overnight at $22^{\circ} \mathrm{C}$. Oxycodone at $0.1,1$, and $10 \mu \mathrm{M}$ concentration in the incubation medium was incubated with one million cells per milliliter for $4 \mathrm{~h}$ with continuous shaking $(100 \mathrm{rpm})$ at $37^{\circ} \mathrm{C}$ in a RED equilibrium dialysis device (Linden Bioscience, MA, USA), using $350 \mu \mathrm{l}$ of $100 \mathrm{mM}$ phosphate buffered saline (PBS) as a receiver side solution. The incubation was conducted in a Heidolph Titramax 1000 incubation cabin (Heidolph Instruments GmbH \& Co. KG, Schwabach, Germany). After incubation, both sides of the dialysis chamber were sampled, and matrices made similar by adding blank cell suspension to buffer sample and buffer to cell suspension sample. Acetonitrile was added to precipitate proteins, samples were centrifuged, and supernatants were analyzed by liquid chromatography-mass spectrometry (LC-MS). The free fraction of oxycodone in the hepatocyte incubation $\left(\mathrm{fu}_{\mathrm{inc}}\right)$ was calculated directly from the peak area ratios measured for each phase: $\mathrm{fu}_{\mathrm{inc}}=$ peak area in receiver sample/peak area in donor sample.

\section{LIOUID CHROMATOGRAPHIC-MASS SPECTROMETRIC ANALYSIS}

The hepatocyte incubation samples were thawed at $22^{\circ} \mathrm{C}$, shaken, and centrifuged for $10 \mathrm{~min}$ at $16,100 \times g$ (Eppendorf 5415D, Eppendorf AG, Hamburg, Germany). The supernatants from 0.1 to $1 \mu \mathrm{M}$ oxycodone incubations were diluted with water to $1: 2$ and those from $10 \mu \mathrm{M}$ oxycodone incubations to 1:5 before analyses. For identification and quantitation of oxycodone and its metabolites, a Waters Acquity ultra-performance liquid chromatographic (UPLC) system with an autosampler, a vacuum degasser, and a column oven was used. The analytical column used was a Waters BEH C18 $(2.1 \mathrm{~mm} \times 50 \mathrm{~mm}, 1.7 \mu \mathrm{m}$; Waters Corporation, Milford, MA, USA). The eluents were $0.02 \%$ ammonia (A, $\mathrm{pH} 9.8$ ) and acetonitrile (B). A gradient elution with a profile $5 \% \mathrm{~B}-5 \%$ $\mathrm{B}-35 \% \mathrm{~B}-85 \% \mathrm{~B}$ in $0,1,3,3.5$ min was employed, followed by column equilibration for $2 \mathrm{~min}$. The flow rate was $0.5 \mathrm{ml} / \mathrm{min}$ and the column oven temperature was $35^{\circ} \mathrm{C}$. Injection volume of $4 \mu \mathrm{l}$ was used. LC/time-of-flight (TOF)-MS data were acquired with a Waters LCT Premier XE TOF-MS equipped with a LockSpray electrospray ionization source. A positive ionization mode of electrospray was used with a capillary voltage of $2800 \mathrm{~V}$ and a cone voltage of $60 \mathrm{~V}$. W-mode ion optics and dynamic range enhancement (DRE) option were used. Aperture 1 voltages of 5 and $65 \mathrm{~V}$ were used to obtain molecular ion data and in-source fragment ion data, respectively. Nitrogen was used as both desolvation and nebulizing gases with flow rates of 800 and $100 \mathrm{l} / \mathrm{h}$, respectively. Desolvation temperature was set to $350^{\circ} \mathrm{C}$ and source gas to $150^{\circ} \mathrm{C}$. The mass range of $\mathrm{m} / z$ 100-750 was acquired with an acquisition time of $150 \mathrm{~ms}$. The mass spectrometer and UPLC system were operated under Micromass MassLynx 4.1 software (Waters Corporation, Milford, MA, USA). Leucine enkephalin was used as lock mass compound $\left([\mathrm{M}+\mathrm{H}]^{+} m / z\right.$ 556.2771) for accurate mass measurements. Metabolites were mined from the data by using Metabolynx XS subroutine of Masslynx-software, employing dealkylation tool and "chemically intelligent" (structure based) mass defect filtering with a $50-\mathrm{mDa}$ tolerance window. The real positives (metabolites) and their identifications were confirmed from the data manually. In quantitation, ion chromatograms with $50 \mathrm{mDa}$ window were used.

Calibration curve with oxycodone was used for quantitation of oxycodone and its metabolites M1-M3 and M5-M8. Correlation coefficient $R^{2}$ was 0.987 for the range $1-2000 \mathrm{nM}$, and back calculated accuracies were $84-103 \%$ in each concentration level, and overall snedecor-precision was $14.2 \%(n=2$ at each concentration). For noroxycodone (M4), a calibration curve with correlation coefficient $R^{2}=0.994$ was obtained for the range $2-2000 \mathrm{nM}$, the back calculated accuracies were $88-107 \%$ in each concentration level, and the overall snedecor-precision was $9.7 \%$.

For analysis of oxycodone samples from hepatocyte binding experiments, a Waters Alliance 2695 HPLC with a column oven and an autosampler was used together with Waters 
XBridge C18 $(2.1 \mathrm{~mm} \times 50 \mathrm{~mm}, 3.5 \mu \mathrm{m})$ column + Phenomenex C18 $(2.0 \mathrm{~mm} \times 4 \mathrm{~mm}, 3.0 \mu \mathrm{m})$ precolumn. The eluents were $0.02 \%$ ammonia (A, pH 9.8) and acetonitrile (B). A gradient elution with profile $5 \% \mathrm{~B}-85 \% \mathrm{~B}-85 \% \mathrm{~B}$ in $0-1-3$ min was employed, followed by column equilibration for $3 \mathrm{~min}$. The flow rate was $0.4 \mathrm{ml} / \mathrm{min}$ and the column oven temperature was $35^{\circ} \mathrm{C}$. Injection volume of $5 \mu \mathrm{l}$ was used. Data was acquired using Waters Quattro Micro triple quadrupole mass spectrometer with $z$-spray electrospray ion source. A capillary voltage of $1400 \mathrm{~V}$ and cone voltage of $28 \mathrm{~V}$ were used. Nitrogen was used as both desolvation and nebulizing gases with flow rates of 800 and $100 \mathrm{l} / \mathrm{h}$, respectively. Desolvation temperature was set to $350^{\circ} \mathrm{C}$ and source gas to $150^{\circ} \mathrm{C}$. Multiple reaction monitoring with positive ion mode was used with transition $\mathrm{m} / z \quad 316>298$ and collision energy of $19 \mathrm{eV}$. Argon was used as a collision gas at $3.8 \times 10^{-3} \mathrm{mbar}$ pressure.

\section{CALCULATION OF IN VITRO CLEARANCE}

The measured oxycodone concentrations $\left(C_{t} ; \mathrm{nM}\right)$ from the duplicate experiment at different sampling times $(t ; \min )$ were fitted into the exponential decay function $\left[C_{\mathrm{t}}=C_{0} \operatorname{EXP}(-k t)\right]$ to get estimates for the concentration at time zero $\left(C_{0} ; \mathrm{nM}\right)$ and elimination rate constant $\left(k ; \min ^{-1}\right)$ using WinNonlin software (version 5.2.1, Pharsight, Mountain View, CA, USA). Apparent in vitro clearance $\left(\mu \mathrm{l} / \mathrm{min} * 10^{6}\right.$ cells) was calculated by multiplying the rate constant with the initial incubation volume $(350 \mu \mathrm{l})$ and dividing the product by 0.35 since there were initially $0.35 \times 10^{6}$ cells in each well. The subsequent samples from the well did not change the ratio of the incubation volume to the number of cells. In vitro clearance based on unbound drug concentration (in vitro CLu) was calculated by diving the apparent clearance by $\mathrm{fu}_{\text {inc }}$.

\section{PREDICTION OF HEPATIC PLASMA CLEARANCE}

The weight and height of the donor was estimated to be the average value for particular age and gender (Kuczmarski et al., 2002; Valentin, 2003). The anatomical and physiological parameters were calculated using the procedures by Johnson et al. (2006) with minor modifications. The body surface area (BSA) was calculated from the weight and height using the equation by Haycock et al. (1978) when the weight was below $15 \mathrm{~kg}$ and the equation from DuBois and DuBois (1916) in other cases. The cardiac output was calculated for children from BSA using the equation 3 in Johnson et al. (2006), for the adult pool the average of male and female values in Valentin (2003) was used. The total hepatic blood flow $\left(\mathrm{Q}_{\mathrm{H}, \mathrm{B}}\right)$ was estimated to be $27 \%$ of cardiac output based on adult data (Valentin, 2003; Johnson et al., 2006). The liver volume (1) was calculated as $0.722 * \mathrm{BSA}^{1.176}$ and it was multiplied with liver density $(1.08 \mathrm{~kg} / \mathrm{l})$ to get liver mass (Johnson et al., 2005). The total number of hepatocytes was obtained by multiplying the liver mass with the recommended hepatocellularity of $99 \times 10^{6}$ cells in gram of liver (Barter et al., 2007). Intrinsic in vivo clearance in the liver based on unbound drug concentration $\left(\mathrm{CLu}_{\text {int, } \mathrm{H}}\right)$ was obtained by multiplying intrinsic in vitro CLu with the total number of hepatocytes. The fraction unbound of oxycodone in plasma ( $\mathrm{fu}$ ) was estimated to be 0.59 (the average from the studies by Leow et al., 1993; Pöyhiä and Seppälä, 1994). The blood to plasma concentration ratio $\left(C_{\mathrm{B}} / C_{\mathrm{P}}\right)$ was estimated to be 1 , but also the value in rats (1.3) in Boström et al. (2006) was tested. The hepatic plasma clearance $\left(\mathrm{CL}_{\mathrm{H}}\right)$ of oxycodone was estimated using the well-stirred model (Yang et al., 2007):

$$
\mathrm{CL}_{\mathrm{H}}=\frac{Q_{\mathrm{H}, \mathrm{B}} \times \mathrm{fu} \times \mathrm{CLu}_{\text {int }, \mathrm{H}}}{Q_{\mathrm{H}, \mathrm{B}}+\frac{\mathrm{fu} \times \mathrm{CLu}_{\text {int }, \mathrm{H}}}{C_{\mathrm{B}} / C_{\mathrm{P}}}}
$$

\section{RESULTS}

\section{FORMATION OF METABOLITES}

Eight oxycodone-related metabolites were observed (Figure 1). All of these metabolites were observed in cells from 4-year-old donor and in pooled adult cells, whereas cells from 3 days, 2 and 5months-old donors contained five to seven metabolites (Table 1). No metabolites specific to a single age group only were observed. The detected metabolites were identified based on the mass spectral accurate mass and fragment ion data and retention times. Due to low abundance of metabolites M1 and M3, fragment ion

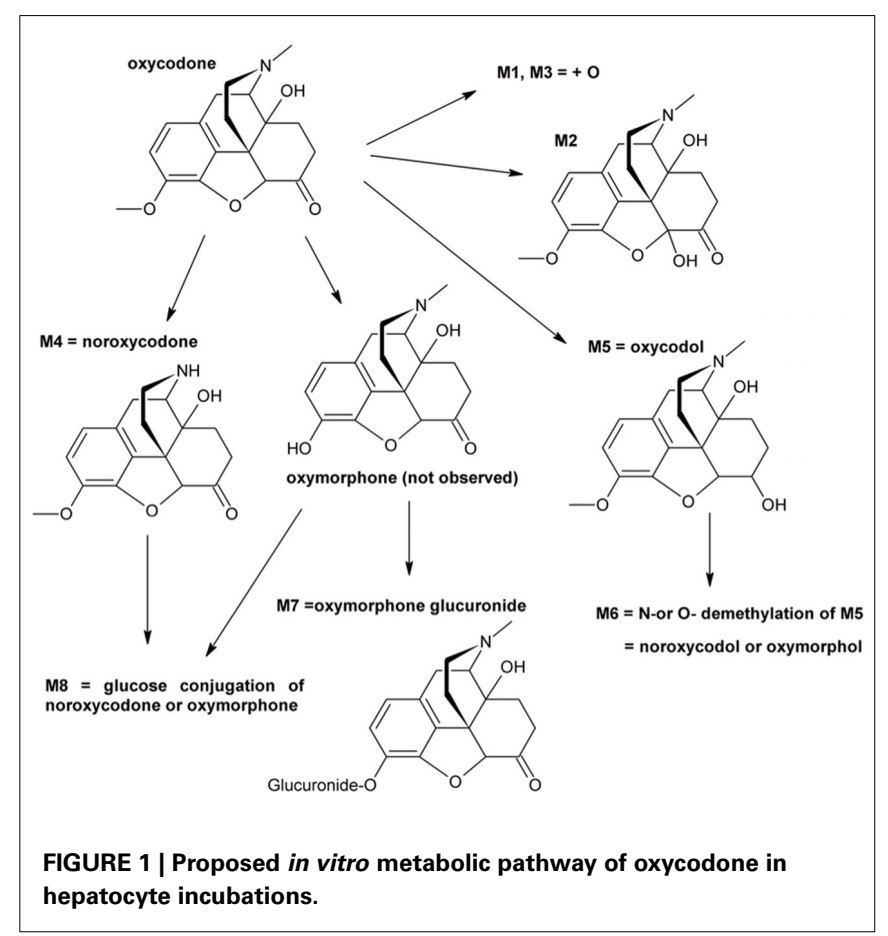

Table 1 | Qualitative metabolite profiles from hepatocyte incubations.

\begin{tabular}{|c|c|c|c|c|c|}
\hline & 3 days & 2 months & 5 months & 4 years & Adult \\
\hline M1 & + & - & - & + & + \\
\hline M2 & + & + & + & + & + \\
\hline M3 & + & + & - & + & + \\
\hline M4 & + & + & + & + & + \\
\hline M5 & + & + & - & + & + \\
\hline M6 & + & + & + & + & + \\
\hline M7 & + & + & + & + & + \\
\hline M8 & - & + & + & + & + \\
\hline
\end{tabular}

Tentative structures of the metabolites are presented in Figure 1. +, Metabolite detected; -, metabolite not detected. 
data was not observed and therefore only tentative identifications as changes in molecular formula with respect to oxycodone were obtained.

Quantitative analytical results revealed that noroxycodone was a major metabolite in all hepatocyte batches (Figure 2). Typically, the parent compound and noroxycodone comprise more than 75\% of drug related material in the last sampling point. Ketoconazole reduced but not completely blocked the conversion of oxycodone to noroxycodone. This suggests that CYP3A is strongly involved in this demethylation reaction in vitro.

\section{IN VITRO CLEARANCE}

The observed free fractions of oxycodone in the hepatocyte incubations $\left(\mathrm{fu}_{\text {inc }}\right.$ ) were $0.93,0.86$, and 0.88 at $0.1,1$, and $10 \mu \mathrm{M}$ oxycodone, respectively. The average value (0.9) was used in the calculation of in vitro clearance based on unbound concentration. In vitro clearance of oxycodone varied in different hepatocyte batches (Figure 3). The highest clearance was observed in the hepatocytes from the 3-day-old donor who had received phenobarbital. In the 3-day, 2-month, and 4-year batches the clearance was fairly constant at different oxycodone concentrations, indicating that there was no clear saturation of metabolism at the highest concentration $(10 \mu \mathrm{M})$. In the adult batch the clearance increased with the concentration for unknown reasons. For the 5-month batch, the clearance could not be estimated at concentrations below $10 \mu \mathrm{M}$ since the $95 \%$ confidence interval for the estimated elimination rate constant included the value zero. CYP3A inhibitor ketoconazole reduced the clearance markedly in the 3-day, 4-year, and adult batches, but not in the 2-month batch (Figure 3).

\section{CORRELATION ANALYSIS}

There was a statistically significant positive correlation (Pearson correlation $0.95, p=0.012, R^{2}=0.91$ ) between the observed in vitro CLu and reported CYP3A4 activity of different cell batches (Table 2). The correlation of in vitro CLu to the reported CYP2D6 is ambiguous because of two different substrates that has been used in hepatocyte lot characterization. However, there seems to be no correlation with observed CLu and CYP2D6 activity (Pearson correlation $\left.-0.17, p=0.78, R^{2}=0.03\right)$.

\section{PREDICTION OF HEPATIC PLASMA CLEARANCE}

In the prediction of hepatic plasma clearance, the intrinsic in vitro clearance (in vitro $\mathrm{CLu}_{\text {int }}$ ) was taken from the incubation with $1 \mu \mathrm{M}$ oxycodone, except for the 5-month batch the value from $10 \mu \mathrm{M}$ oxycodone was used (Table 3 ). The predicted hepatic clearance was in most cases markedly lower than the median total plasma clearance observed in pharmacokinetic studies, being
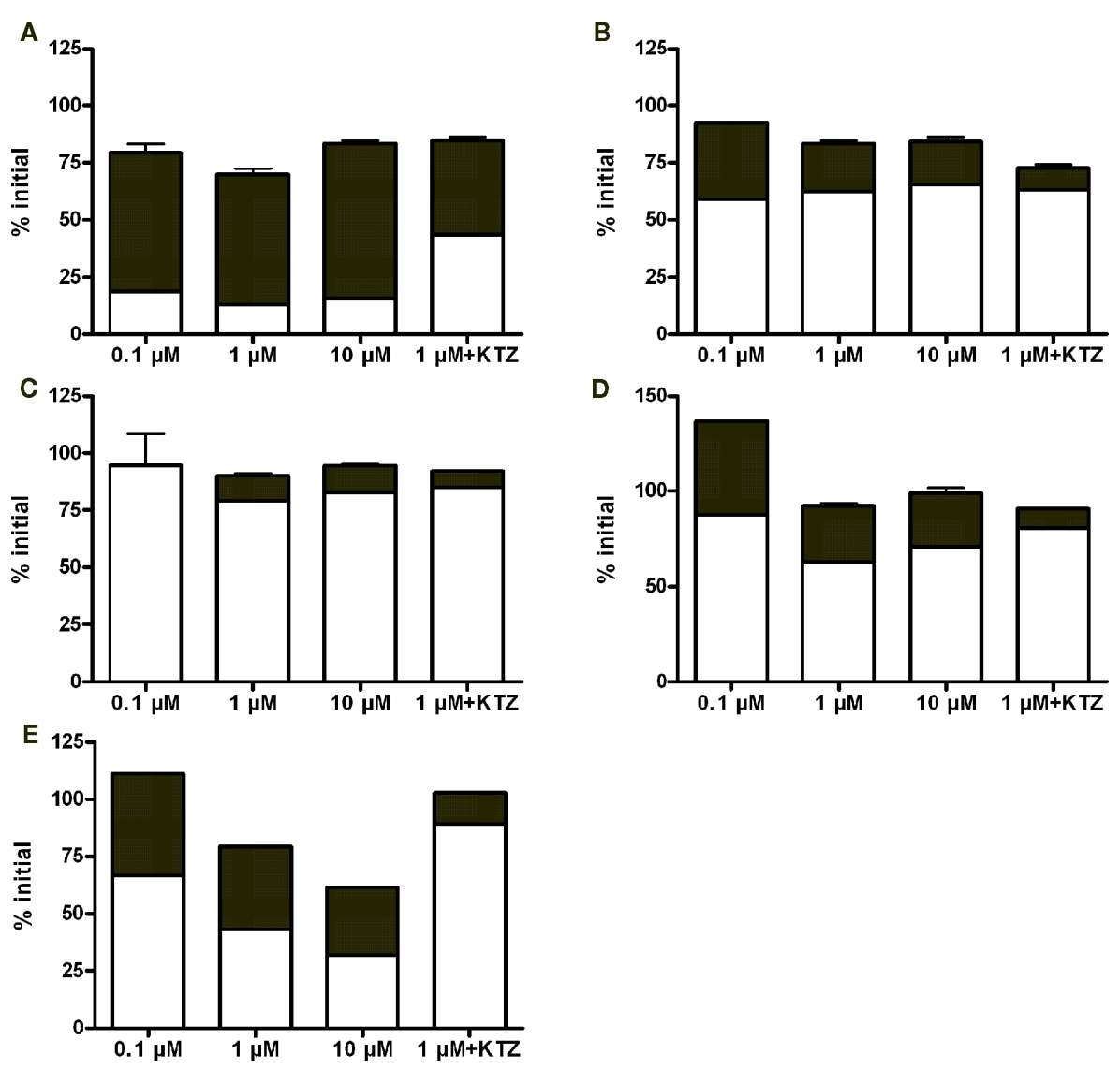

FIGURE 2 | Fractional concentrations of oxycodone (white bars) and noroxycodone (black bars) at the last time point (4 $\mathrm{h}$ ) in hepatocyte incubations from 3 day (A), $\mathbf{2}$ month (B), 5 month (C), 4 year (D), and adult (E) donors. Bars represent mean \pm SD (single experiment in duplicate). KTZ, ketoconazole. 
either close to the minimum total clearance ( 2 months and adult pool) or even lower than that ( 5 months and 4 years). The exception was the batch from the 3-day-old donor. In this case the predicted hepatic clearance was slightly higher than the observed median total clearance. When the calculations for the adult were made using in vitro CLu from the incubation with $10 \mu \mathrm{M}$ oxycodone $\left[6.6 \mu \mathrm{l} /\left(\mathrm{min} * 10^{6}\right.\right.$ cells $\left.)\right]$, in vivo $\mathrm{CLu}_{\mathrm{int}, \mathrm{H}}$ of $1000 \mathrm{ml} / \mathrm{min}$ and predicted hepatic clearance of $6.5 \mathrm{ml} /(\mathrm{min} * \mathrm{~kg})$ were obtained. The latter is one half of the observed median total clearance.

\section{DISCUSSION}

In this study, we evaluated an in vitro to in vivo extrapolation method to estimate the maturation of oxycodone metabolism in humans. Generally, the ontogeny of hepatic clearance pathways has been studied actively and several methods have been developed for

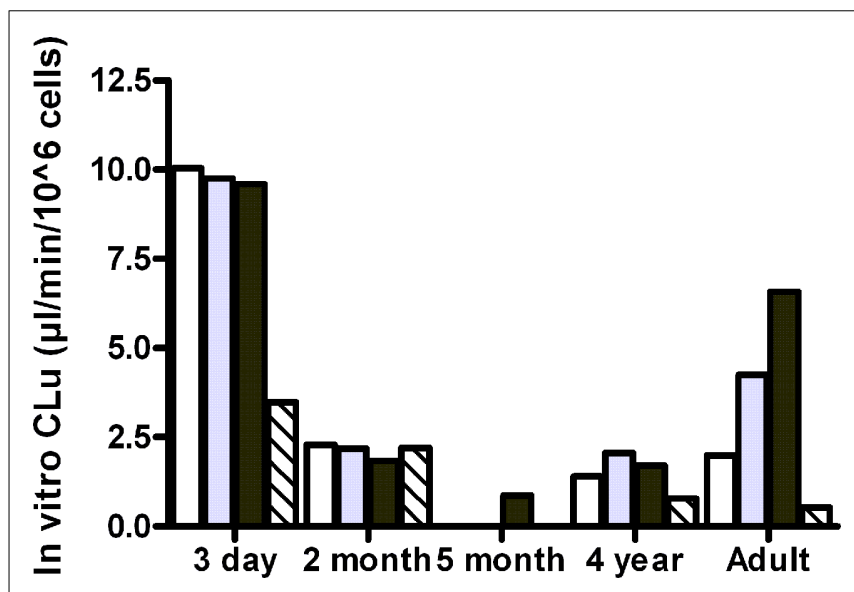

FIGURE 3 | In vitro clearance of oxycodone based on unbound concentration (in vitro $\mathrm{CLu}$ ) in cryopreserved human hepatocyte at $0.1 \mu \mathrm{M}$ (white bars), $1 \mu \mathrm{M}$ (gray bars), $10 \mu \mathrm{M}$ (black bars), or $1 \mu \mathrm{M}$ in the presence of ketoconazole (hatched bars). the prediction of hepatic clearance in infants and children (Alcorn and McNamara, 2002; Edginton et al., 2006; Johnson et al., 2006). In these methods the activity of each metabolic enzyme vs. age is described mathematically based on the available data on the expression and activity (in vitro and in vivo clearance of various substrates) of the enzyme. The input data needed are either the pharmacokinetic parameters of the drug in adults or MichaelisMenten parameters of the involved enzymes from in vitro studies. Our approach was to utilize hepatocyte preparations from different age groups to study age-dependent metabolism directly in vitro.

The metabolism in different age groups was qualitatively fairly similar (Figure 1; Table 1). Worth noticing is that the known oxycodone metabolites oxymorphone and noroxymorphone (Lalovic et al., 2004, 2006) were not detected in the samples, even thought the oxymorphone was detected as a glucuronide conjugated form (M7), and possibly also in M6 and M8, for which the demethylation site remained unclear. One reason for this may be the observed poor stability of these compounds in incubation medium since the prepared oxymorphone and noroxymorphone standard samples were found to degradate very rapidly in used autosampler conditions $(2 \mu \mathrm{M}$ concentration both the substances disappeared completely in $12 \mathrm{~h}$ ).

The importance of CYP3A in the metabolism of oxycodone in the hepatocytes was supported by the data. Noroxycodone, known to be formed by CYP3A4 and CYP3A5 (Lalovic et al., 2004), was a major metabolite in all batches. CYP3A inhibitor ketoconazole inhibited the metabolism of oxycodone and formation of noroxycodone in most cases. There was also a positive correlation between oxycodone clearance and CYP3A4 activity but not CYP2D6 activity. The CYP3A-mediated noroxycodone formation has been shown to be eightfold with respect to CYP2D6mediated oxymorphone formation in human liver microsomes (Lalovic et al., 2004). Also, urinary excretion data have revealed that CYP3A-mediated $N$-demethylation is the principal metabolic pathway in adults (Kirvelä et al., 1996; Lalovic et al., 2006).

Table 2 | Experimentally measured in vitro clearance of oxycodone and reported CYP3A4 and CYP2D6 activities in hepatocyte batches used in the study.

\begin{tabular}{|c|c|c|c|c|c|}
\hline Donor age & 3 days & 2 months & 5 months & 4 years & Adult pool \\
\hline In vitro $\mathrm{CLu}$ & 9.8 & 2.2 & 0.9 & 2.1 & 4.2 \\
\hline CYP3A4 activity ${ }^{1}$ & 790 & 212 & 63 & 310 & $210-280^{2}$ \\
\hline Activity vs. adult batch & 3.2 & 0.9 & 0.3 & 1.3 & $1.0^{3}$ \\
\hline CYP2D6 activity ${ }^{4}$ & 15 & - & 6.7 & 30 & - \\
\hline CYP2D6 activity ${ }^{5}$ & & 29 & & & 15.5 \\
\hline Activity vs. adult batch & & 1.9 & & & 1.0 \\
\hline
\end{tabular}

In vitro CLu ( $\mu \mathrm{l} / \mathrm{min} / 10^{6}$ cells) obtained from the incubation with $1 \mu \mathrm{M}$ oxycodone, except for the 5-month batch from $10 \mu \mathrm{M}$ oxycodone. All the CYP activities

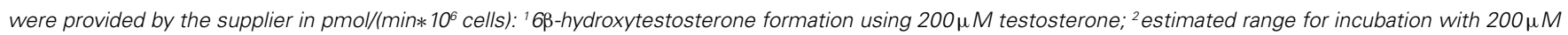

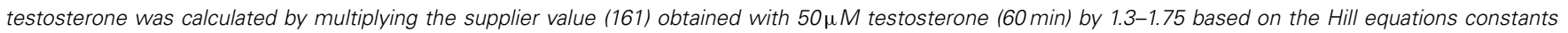

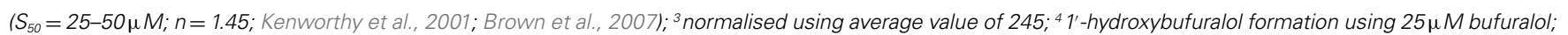
${ }^{5}$ dextrorphan formation using $15 \mu \mathrm{M}$ dextromethorphan (2 month) or $8 \mu \mathrm{M}$ dextromethorphan (adult). 
Table 3 | Prediction of hepatic plasma clearance of oxycodone using in vitro clearance in hepatocytes and physiological parameters.

\begin{tabular}{|c|c|c|c|c|c|}
\hline Donor age & 3 days & 2 months & 5 months & 4 years & Adult pool \\
\hline Gender & Female & Male & Female & Female & Female + male \\
\hline Weight (kg) & 3.4 & 5.3 & 6.7 & 16 & 67 \\
\hline Body surface area $\left(\mathrm{m}^{2}\right)$ & 0.22 & 0.3 & 0.35 & 0.66 & 1.78 \\
\hline Cardiac output (ml/min) & 510 & 710 & 840 & 1980 & 6200 \\
\hline Total hepatocytes $\left(10^{6}\right)$ & 13,000 & 19,000 & 23,000 & 48,000 & 152,000 \\
\hline In vitro $\mathrm{CLu}_{\mathrm{int}}{ }^{1}\left[\mu \mathrm{l} /\left(\mathrm{min}^{*} 10^{6}\right.\right.$ cells $\left.)\right]$ & 9.8 & 2.2 & 0.9 & 2.1 & 4.2 \\
\hline In vivo $\mathrm{CLu}$ int, $\mathrm{H}(\mathrm{ml} / \mathrm{min})$ & 127 & 42 & 20 & 99 & 644 \\
\hline \multicolumn{6}{|l|}{ Predicted $\mathrm{Cl}_{\mathrm{H}}$} \\
\hline $\mathrm{ml} / \mathrm{min}$ & 49 & 22 & 11 & 53 & 310 \\
\hline
\end{tabular}

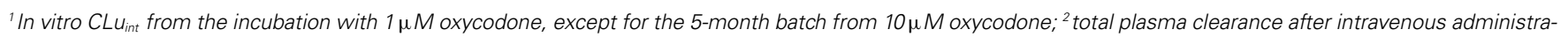

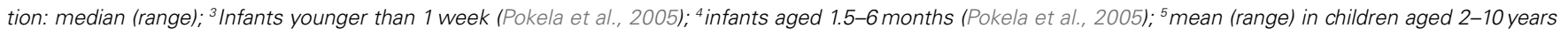
(Olkkola et al., 1994; Kokki et al., 2004); 'healthy volunteers and cancer patients aged 19-71 years (Pöyhiä et al., 1991; Leow et al., 1992; Takala et al., 1997).

In vitro clearance of oxycodone in the hepatocytes was used to predict hepatic plasma clearance. In the adults, the median hepatic plasma clearance is ca. $11 \mathrm{ml} /(\mathrm{min} * \mathrm{~kg})$ based on the findings that total plasma clearance is ca. $12 \mathrm{ml} /(\min * \mathrm{~kg}$; Table 3$)$ and ca. $10 \%$ of the dose is excreted unchanged in urine (Pöyhiä et al., 1992; Kirvelä et al., 1996; Lalovic et al., 2006). The predicted hepatic clearance using pooled adult hepatocytes with 1 and $10 \mu \mathrm{M}$ oxycodone were ca. 40 and $60 \%$ of the observed median value. Earlier, Lalovic et al. (2004) reported a mean intrinsic clearance of $12 \mu \mathrm{l} / \mathrm{min} / \mathrm{mg}$ microsomal protein and negligible binding in human liver hepatocytes. Thus, an intrinsic in vivo clearance of 590 or $740 \mathrm{ml} / \mathrm{min}$ is extrapolated (methods of Barter et al., 2007; Hallifax and Houston, 2009); which are close to our value at $1 \mu \mathrm{M}$ oxycodone (644 ml/min; Table 3 ).

The predicted plasma clearances obtained with hepatocytes from individual infants and children were significantly lower than the observed median total plasma clearance, except for the 3-dayold donor. The hepatic plasma clearance of oxycodone in infants and children is not known accurately since the urinary excretion of oxycodone and its metabolites has not been reported. However, plasma concentrations of oxycodone, noroxycodone, and oxymorphone have been determined in children aged $0.5-8$ years after buccal and sublingual administration (Kokki et al., 2006). In these children the AUC ratio of noroxycodone to oxycodone was approximately as high and AUC ratio of oxymorphone to oxycodone as low as in adults after oral administration. This suggests that in these children the metabolic clearance and especially the role of CYP3A is as important as in adults. There are no pharmacokinetic data on the metabolism of oxycodone in infants aged below

\section{REFERENCES}

Alcorn, J., and McNamara, P. J. (2002). Ontogeny of hepatic and renal systemic clearance pathways in infants: part
II. Clin. Pharmacokinet. 41, 1077-1094.

Barter, Z. E., Bayliss, M. K., Beaune, P. H., Boobis, A. R., Carlile, D. J., Edwards, R. J., Houston, J. B., Lake,
0.5 years since only plasma concentrations of oxycodone have been determined (Pokela et al., 2005).

Under-prediction of hepatic clearance based on in vitro data from hepatocyte and microsome incubations is common (Hallifax and Houston, 2009; Foster et al., 2011). Compounds with low metabolic clearance in vitro produce often poor in vitro in vivo extrapolations based on parent compound metabolic disappearance (Chiba et al., 2009) partly because active uptake to liver can affect in vivo clearance but is not evident from typical in vitro stability results (Soars et al., 2007). Oxycodone transport across blood-brain barrier is partly dependent on some organic cation transporter (Okura et al., 2008) and P-glycoprotein can also transport oxycodone (Hassan et al., 2007). Therefore, active uptake to liver can cause the observed under-prediction of oxycodone clearance since only rate of metabolism was measured in vitro.

In the present study, cryopreserved hepatocytes from different age groups were used to study the maturation of the drug metabolism. These studies reveal that similar metabolites are formed in children and adults. For oxycodone, this is important information since knowledge on in vivo metabolite profiles in youngest infants is missing (Pokela et al., 2005). The data suggests that the major determinant of oxycodone clearance in all age groups is CYP3A activity. Therefore, oxycodone clearance may follow general CYP3A ontogeny and possible inter-individual variability within age groups.

\section{ACKNOWLEDGMENTS}

We would like to thank PhD Ilpo Rasanen for helpful discussions.
B. G., Lipscomb, J. C., Pelkonen, O. R., Tucker, G. T., and RostamiHodjegan, A. (2007). Scaling factors for the extrapolation of in vivo metabolic drug clearance from in vitro data: reaching a consensus on values of human microsomal protein and hepatocellularity per gram of liver. Curr. Drug Metab. 8, 33-45. 
Boström, E., Simonsson, U. S., and Hammarlund-Udenaes, M. (2006). In vivo blood-brain barrier transport of oxycodone in the rat: indications for active influx and implications for pharmacokinetics/pharmacodynamics. Drug Metab. Dispos. 34, 1624-1631.

Brown, H. S., Griffin, M., and Houston, J. B. (2007). Evaluation of cryopreserved human hepatocytes as an alternative in vitro system to microsomes for the prediction of metabolic clearance. Drug Metab. Dispos. 35, 293-301.

Chiba, M., Ishii, Y., and Sugiyama, Y. (2009). Prediction of hepatic clearance in human from in vitro data for successful drug development. AAPS J. 11, 262-276.

DuBois, D., and DuBois, E. F. (1916). A formula to estimate the approximate surface area if height and weight be known. Arch. Intern. Med. 17, 863-871.

Edginton, A. N., Schmitt, W., Voith, B., and Willmann, S. (2006). A mechanistic approach for the scaling of clearance in children. Clin. Pharmacokinet. 45, 683-704.

El-Tahtawy, A., Kokki, H., and Reidenberg, B. E. (2006). Population pharmacokinetics of oxycodone in children 6 months to 7 years old. J. Clin. Pharmacol. 46, 433-442.

Falk, E. (1917). Eukodal, ein neues narkotikum. Munch. Med. Wochenschr. 20, 381-384.

FDA Guidance for Industry. (1998). General Considerations for Pediatric Pharmacokinetic Studies for Drugs and Biological Products. Rockville: Food and Drug Administration.

Foster, J. A., Houston, J. B., and Hallifax, D. (2011). Comparison of intrinsic clearances in human liver microsomes and suspended hepatocytes from the same donor livers: clearance-dependent relationship and implications for prediction of in vivo clearance. Xenobiotica 41, 124-136.

Hallifax, D., and Houston, J. B. (2009). Methodological uncertainty in quantitative prediction of human hepatic clearance from in vitro experimental systems. Curr. Drug Metab. 10, 307-321.

Hassan, H. E., Myers, A. L., Lee, I. J., Coop, A., and Eddington, N. D. (2007). Oxycodone induces overexpression of P-glycoprotein
(ABCB1) and affects paclitaxel's tissue distribution in Sprague Dawley rats. J. Pharm. Sci. 96, 2494-2506.

Haycock, G. B., Schwartz, G. J., and Wisotsky, D. H. (1978). Geometric method for measuring body surface area: a height-weight formula validated in infants, children, and adults. J. Pediatr. 93, 62-66.

Heiskanen, T., Olkkola, K. T., and Kalso, E. (1998). Effects of blocking CYP2D6 on the pharmacokinetics and pharmacodynamics of oxycodone. Clin. Pharmacol. Ther. 64, 603-611.

International Narcotics Control Board. (2010). Narcotic Drugs: Estimated World Requirements for 2010; Statistics for 2008. New York: United Nations.

Johnson, T. N., Rostami-Hodjegan, A., and Tucker, G. T. (2006). Prediction of the clearance of eleven drugs and associated variability in neonates, infants and children. Clin. Pharmacokinet. 45, 931-956.

Johnson, T. N., Tucker, G. T., Tanner, M. S., and Rostami-Hodjegan, A. (2005). Changes in liver volume from birth to adulthood: a meta-analysis. Liver Transpl. 11, 1481-1493.

Kenworthy, K. E., Clarke, S. E., Andrews, J., and Houston, J. B. (2001). Multisite kinetic models for CYP3A4: simultaneous activation and inhibition of diazepam and testosterone metabolism. Drug Metab. Dispos. 29 1644-1651.

Kirvelä, M., Lindgren, L., Seppälä, T., and Olkkola, K. T. (1996). The pharmacokinetics of oxycodone in uremic patients undergoing renal transplantation. J. Clin. Anesth. 8, 13-18.

Kokki, H., Rasanen, I., Laisalmi, M., Lehtola, S., Ranta, V. P., Vanamo, K., and Ojanperä, I. (2006). Comparison of oxycodone pharmacokinetics after buccal and sublingual administration in children. Clin. Pharmacokinet. 45, 745-754.

Kokki, H., Rasanen, I., Reinikainen, M., Suhonen, P., Vanamo, K., and Ojanperä, I. (2004). Pharmacokinetics of oxycodone after intravenous, buccal, intramuscular and gastric administration in children. Clin. Pharmacokinet. 43, 613-622.

Kuczmarski, R. J., Ogden, C. L., and Guo, S. S., Grummer-Strawn, L. M., Flegal, K. M., Mei, Z., Wei, R., Curtin, L. R., Roche, A. F., and Johnson, C.
L. (2002). 2000 CDC growth charts for the United States: methods and development. Vital Health Stat. 11, 1-190.

Lalovic, B., Kharasch, E., Hoffer, C. Risler, L., Liu-Chen, L. Y., and Shen, D. D. (2006). Pharmacokinetics and pharmacodynamics of oral oxycodone in healthy human subjects: role of circulating active metabolites. Clin. Pharmacol. Ther. 79, 461-479.

Lalovic, B., Phillips, B., Risler, L. L., Howald, W., and Shen, D. D (2004). Quantitative contribution of CYP2D6 and CYP3A to oxycodone metabolism in human liver and intestinal microsomes. Drug Metab. Dispos. 32, 447-454.

Lemberg, K. K., Heiskanen, T. E., Neuvonen, M., Kontinen, V. K., Neuvonen, P. J., Dahl, M. L., and Kalso, E. A. (2010). Does co-administration of paroxetine change oxycodone analgesia: an interaction study in chronic pain patients. Scand. J. Pain 1, 24-33.

Leow, K. P., Smith, M. T., Williams, B., and Cramond, T. (1992). Singledose and steady-state pharmacokinetics and pharmacodynamics of oxycodone in patients with cancer. Clin. Pharmacol. Ther. 52, 487-495.

Leow, K. P., Wright, A. W., Cramond, T. and Smith, M. T. (1993). Determination of the serum protein binding of oxycodone and morphine using ultrafiltration. Ther. Drug Monit. 15, 440-447.

Okura, T., Hattori, A., Takano, Y. Sato, T., Hammarlund-Udenaes, M. Terasaki, T., and Deguchi, Y. (2008). Involvement of the pyrilamine transporter, a putative organic cation transporter, in blood-brain barrier transport of oxycodone. Drug Metab. Dispos. 36, 2005-2013.

Olkkola, K. T., Hamunen, K., Seppälä, T. and Maunuksela, E. L. (1994). Pharmacokinetics and ventilatory effects of intravenous oxycodone in postoperative children. Br. J. Clin. Pharmacol. 38, 71-76.

Pokela, M. L., Anttila, E., Seppälä, T., and Olkkola, K. T. (2005). Marked variation in oxycodone pharmacokinetics in infants. Paediatr. Anaesth. 15, 560-565.

Pöyhiä, R., Olkkola, K. T., Seppälä, T. and Kalso, E. (1991). The pharmacokinetics of oxycodone after intravenous injection in adults. $\mathrm{Br}$. J. Clin. Pharmacol. 32, 516-518.
Pöyhiä, R., and Seppälä, T. (1994). Liposolubility and protein binding of oxycodone in vitro. Pharmacol. Toxicol. 74, 23-27.

Pöyhiä, R., Seppälä, T., Olkkola, K. T., and Kalso, E. (1992). The pharmacokinetics and metabolism of oxycodone after intramuscular and oral administration to healthy subjects. Br. J. Clin. Pharmacol. 33, 617-621.

Soars, M. G., Grime, K., Sproston, J. L., Webborn, P. J., and Riley, R. J. (2007). Use of hepatocytes to assess the contribution of hepatic uptake to clearance in vivo. Drug Metab. Dispos. 35, 859-865.

Takala, A., Kaasalainen, V., Seppälä, T., Kalso, E., and Olkkola, K. T. (1997). Pharmacokinetic comparison of intravenous and intranasal administration of oxycodone. Acta Anaesthesiol. Scand. 41, 309-312.

Valentin, J. (ed.). (2003). Basic anatomical and physiological data for use in radiological protection: reference values. Ann. ICRP Publication 89, 1-265.

Yang, J., Jamei, M., Yeo, K. R., RostamiHodjegan, A., and Tucker, G. T. (2007). Misuse of the well-stirred model of hepatic drug clearance. Drug Metab. Dispos. 35, 501-502.

Conflict of Interest Statement: An unrestricted grant was received from Mundipharma Oy, Vantaa, Finland, to cover the costs of the chemicals.

Received: 19 October 2011; accepted: 16 December 2011; published online: 05 January 2012.

Citation: Korjamo T, Tolonen A, Ranta $V$-P, Turpeinen $M$ and Kokki H (2012) Metabolism of oxycodone in human hepatocytes from different age groups and prediction of hepatic plasma clearance. Front. Pharmacol. 2:87. doi: 10.3389/fphar.2011.00087

This article was submitted to Frontiers in Drug Metabolism and Transport, a specialty of Frontiers in Pharmacology. Copyright (c) 2012 Korjamo, Tolonen, Ranta, Turpeinen and Kokki. This is an open-access article distributed under the terms of the Creative Commons Attribution Non Commercial License, which permits non-commercial use, distribution, and reproduction in other forums, provided the original authors and source are credited. 